\title{
Formulation development and evaluation of medroxyprogesterone acetate injectable suspension
}

\author{
Dr. M. Sunitha Reddy*, B. Samatha \\ Department of pharmaceutics, Centre of pharmaceutical sciences, IST, JNTU, Hyderabad, India
}

\begin{abstract}
The aim of the present work is to develop sustained release parenteral drug delivery system of contraceptive drug i., e medroxyprogesterone acetate. The formulation was prepared by sterile combining of API and excipient powders by rapid stirring method. Different excipients used in the formulation are PEG 3350, Poloxamer -188, Polysorbate-80, Benzyl alcohol and Sodium chloride. The prepared parenteral suspension was evaluated for all official parameters like sedimentation volume, measurement of zeta potential, pH, viscosity, osmolality, dissolution, assay, particle size determination, and stability studies. The dissolution profile was found to be more when compared with the innovator formulation. Stability studies were also conducted at $25^{\circ} \mathrm{C} / 60 \% \mathrm{RH}$ and $40^{\circ} \mathrm{C} / 75 \% \mathrm{RH}$ conditions up to $3 \mathrm{M}$.All the parameters evaluated are within the specified limits.
\end{abstract}

Keywords: Medroxy progesterone acetate, PEG 3350, Poloxamer-188, injectable suspension.

Article Info: Received 31 Oct 2018; Review Completed 10 Dec 2018; Accepted 13 Dec 2018; Available online 15 Dec 2018

\section{Cite this article as:}

Sunitha Reddy M, Samatha B, Formulation development and evaluation of medroxyprogesterone acetate injectable suspension, Journal of Drug Delivery and Therapeutics. 2018; 8(6-s):298-303

DOI: http://dx.doi.org/10.22270/jddt.v8i6-s.2146

口. Address for Correspondence:

Dr. M. Sunitha Reddy, M. pharm, Ph.D., Assistant Professor, BOS chairperson, Centre of pharmaceutical sciences, IST, JNTUH, Hyderabad, India

\section{INTRODUCTION}

The parenteral route is the most preeminent and common form of drug delivery system. This system generally avoids the drug related problems which were associated with the oral route are acid degradation, hepatic metabolism (first pass effect), enzymatic action in the intestine, unpredictable pharmacokinetic parameters (solubility, absorption, distribution etc..,). Site specific drug delivery can also be achieved by this route ${ }^{1}$.

To overcome the loopholes various approaches like microemulsions ${ }^{2}$, liposomes, solid dispersion techniques, cubosomes and complexation with cyclodextrins are adopted. Though there reasonable success has been achieved employing these techniques but still some of the draw backs were there such as poor stability, low drug loading capacity, increased toxicity, complex manufacturing method ${ }^{3}$.

\section{Depot systems}

A depot is an injection, either administered intramuscularly or subcutaneously which usually deposits the drug at the site of injection as a mass, from which it gets absorbed and distributed to the tissue which surrounds it. This system usually releases the active compound in a consistent manner over a long period. The main advantage of long-acting depot injection includes decreased dosing frequency which in turns ISSN: 2250-1177 increases the patient compliance; more consistent serum concentration can be gained 4 .

Parenteral suspensions are thermodynamically unstable heterogeneous systems in which the insoluble drug particles are dispersed or suspended in the dispersion medium (such as aqueous or non-aqueous vehicles). Particle size should be less than $5 \mu \mathrm{m}^{5}$. Medroxy progesterone structurally resembles progesterone and it is widely used as contraceptive or hormone replacement therapy ${ }^{6}$. The overall aim of the present work was to formulate the parenteral depot suspension and illustrate the particle size, $\mathrm{pH}$, in- vitro drug release profile and stability parameters with that of innovator product.

\section{MATERIALS AND METHODS}

Medroxy progesterone acetate was obtained from Crystal pharma as a gift sample. Polyethylene glycol 3350 was purchased from Sigma Aldrich, USA. Polysorbaten80, poloxamer-188, benzyl alcohol, sodium chloride and sodium hydroxide were obtained from Merck chemicals Ltd., Mumbai. All other chemicals and reagents used were of analytical reagent (AR) grade. 


\section{Preformulation studies ${ }^{7}$}

Preformulation is the study that yields necessary information related to physicochemical properties of drug candidate in order to develop safe, effective and stable dosage form. This study includes

- Physical characterization of API

- Solubility of API

- Drug-excipient compatibility study

\section{Preparation of aqueous suspension}

Aqueous suspension of medroxy progesterone acetate was prepared by rapid stirring method. Accurately weighed quantity of PEG-3350, poloxamer 188, Polysorbate 80, benzyl alcohol and sodium chloride was dissolved in Milli-Q water by continuous stirring. The API was added during stirring condition, at least for 45 minutes $^{8}$. The formulation design for medroxy progesterone acetate suspension is represented (Table No. 1).

Table 1: Formulation design of Medroxy progesterone acetate injectable suspension

\begin{tabular}{|c|c|c|c|c|c|c|c|c|c|c|}
\hline S.No & $\begin{array}{c}\text { Name of the ingredients } \\
(\mathrm{mg} / \mathrm{mL})\end{array}$ & F1 & F2 & F3 & F4 & F5 & F6 & F7 & F8 & F9 \\
\hline 1. & $\begin{array}{l}\text { Medroxy progesterone } \\
\text { Acetate }\end{array}$ & 150 & 150 & 150 & 150 & 150 & 150 & 150 & 150 & 150 \\
\hline 2. & Polyethylene glycol 3350 & 27.1 & 26.9 & 28.5 & - & - & - & 28.1 & 27.7 & 26.9 \\
\hline 3. & Poloxomer 188 & - & - & - & 1.0 & 1.50 & 2.0 & 0.80 & 1.20 & 2.0 \\
\hline 4. & Polysorbate 80 & 2.41 & 2.41 & 2.41 & 2.41 & 2.41 & 2.41 & 2.41 & 2.41 & 2.41 \\
\hline 5. & Sodium chloride & 8.68 & 8.68 & 8.68 & 8.68 & 8.68 & 8.68 & 8.68 & 8.68 & 8.68 \\
\hline 6. & Benzyl alcohol & 4.45 & 7.10 & 8.88 & 5.6 & 6.90 & 9.16 & 7.5 & 6.80 & 8.88 \\
\hline 7. & Sodium hydroxide $(0.1 \mathrm{~N})$ & q.s & q.s & q.s & q.s & q.s & q.s & q.s & q.s & q.s \\
\hline 8. & Hydrochloric acid $(0.1 \mathrm{~N})$ & q.s & q.s & q.s & q.s & q.s & q.s & q.s & q.s & q.s \\
\hline 9. & Water for injection & q.s & q.s & q.s & q.s & q.s & q.s & q.s & q.s & q.s \\
\hline
\end{tabular}

\section{In-vitro Dissolution studies}

In-vitro dissolution test was carried out by using USP type-IV apparatus (Flow through cell) ${ }^{9} .900 \mathrm{~mL}$ of $\mathrm{pH} 7.4$ phosphate buffer and $0.50 \%$ SLS was used as dissolution medium. Basket was rotated at $50 \mathrm{rpm}$ and temperature was maintained at $37 \pm 0.5^{\circ} \mathrm{C}$. Sampling was done at regular intervals and they are analysed by HPLC.

\section{Physical characterization of suspension}

- $\quad$ Particle size and zeta potential

- $\mathrm{pH}$

- Osmolality

- Viscosity

\section{RESULTS AND DISCUSSION}

Table 2: Physical characterization of API

\begin{tabular}{lll}
\hline S.No & Description & Results \\
\hline 1. & Colour & White \\
2. & Appearance & Crystalline powder \\
3. & Taste & Bitter \\
4. & odor & Odorless \\
\hline
\end{tabular}

\section{X-Ray diffraction study}

Sharp peaks were observed in the X-Ray diffractogram indicates crystalline nature of API.

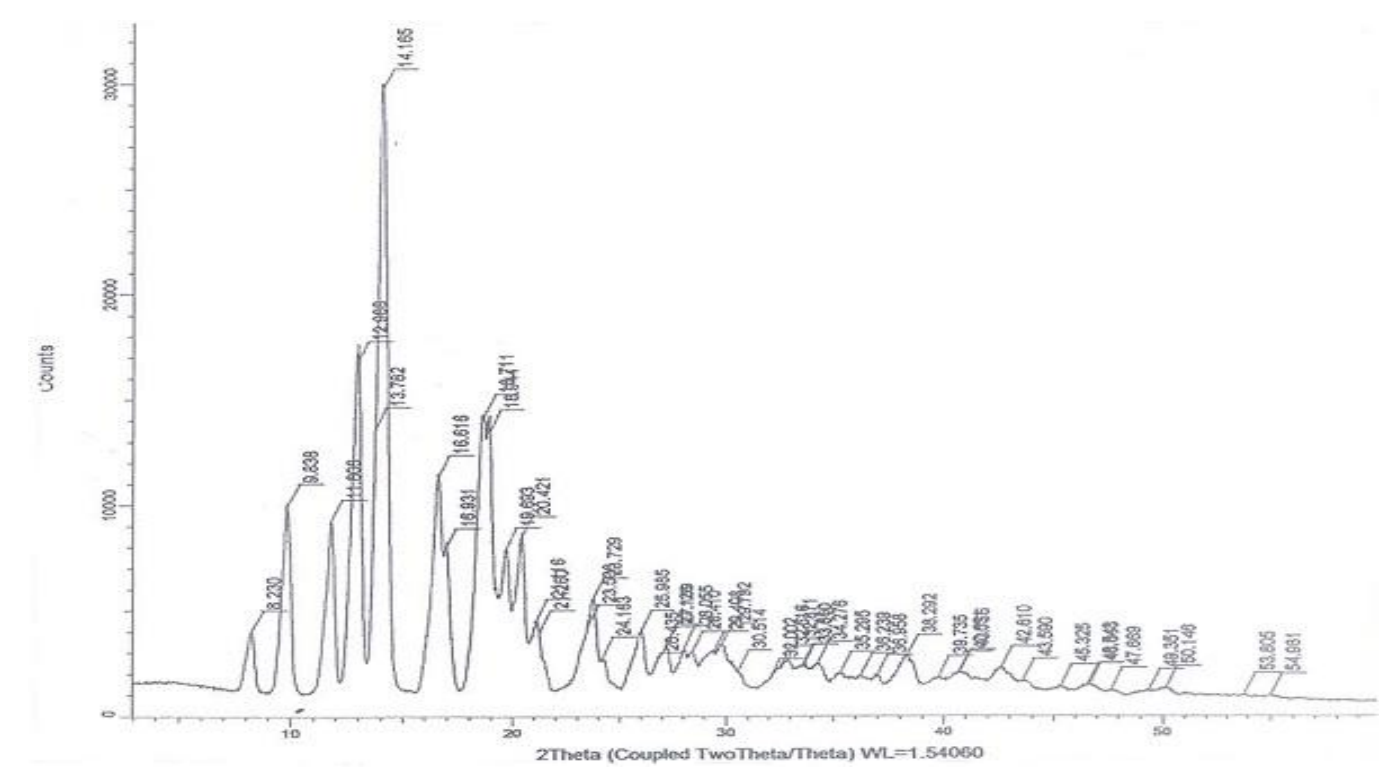

Figure 1: XRD of Medroxy progesterone acetate 
Table 3: Solubility of API in different media

\begin{tabular}{lc}
\hline Solvent system & Solubility $\mathbf{( m g / m L )}$ \\
\hline Purified water & 0.0033 \\
0.1 N Hcl & 0.0003 \\
pH 4.5 acetate buffer & 0.0004 \\
pH 6.8 phosphate buffer & 0.0002 \\
pH 7.4 phosphate buffer & 0.0014 \\
Water + 0.5 \% SLS & 0.355 \\
0.1 N Hcl + 0.5 \% SLS & 0.352 \\
pH 4.5 acetate buffer + 0.5 \% SLS & 0.383 \\
pH 6.8 phosphate buffer + 0.5 \% SLS & 0.329 \\
pH 7.4 phosphate buffer + 0.5 \% SLS & 0.449 \\
\hline
\end{tabular}

\section{Drug-Excipient compatibility studies by FTIR}

FTIR method was adopted to study the compatibility with drug and excipients. Medroxy progesterone acetate shows characteristics absorption bands at $3424.31 \mathrm{~cm}^{-1}$ for $\mathrm{O}-\mathrm{H}$,
$2947.26 \mathrm{~cm}^{-1}$ for $\mathrm{C}-\mathrm{H}$ stretching, $1731.79 \mathrm{~cm}-1$ for $\mathrm{C}=0$ stretching and $1410.27 \mathrm{~cm}^{-1} \mathrm{C}=\mathrm{C}$ bending. There were no extra peaks observed in the spectrum when API in combination with excipient. A figure $1,2 \& 3$ represents the compatibility between drug and the excipients mixture.

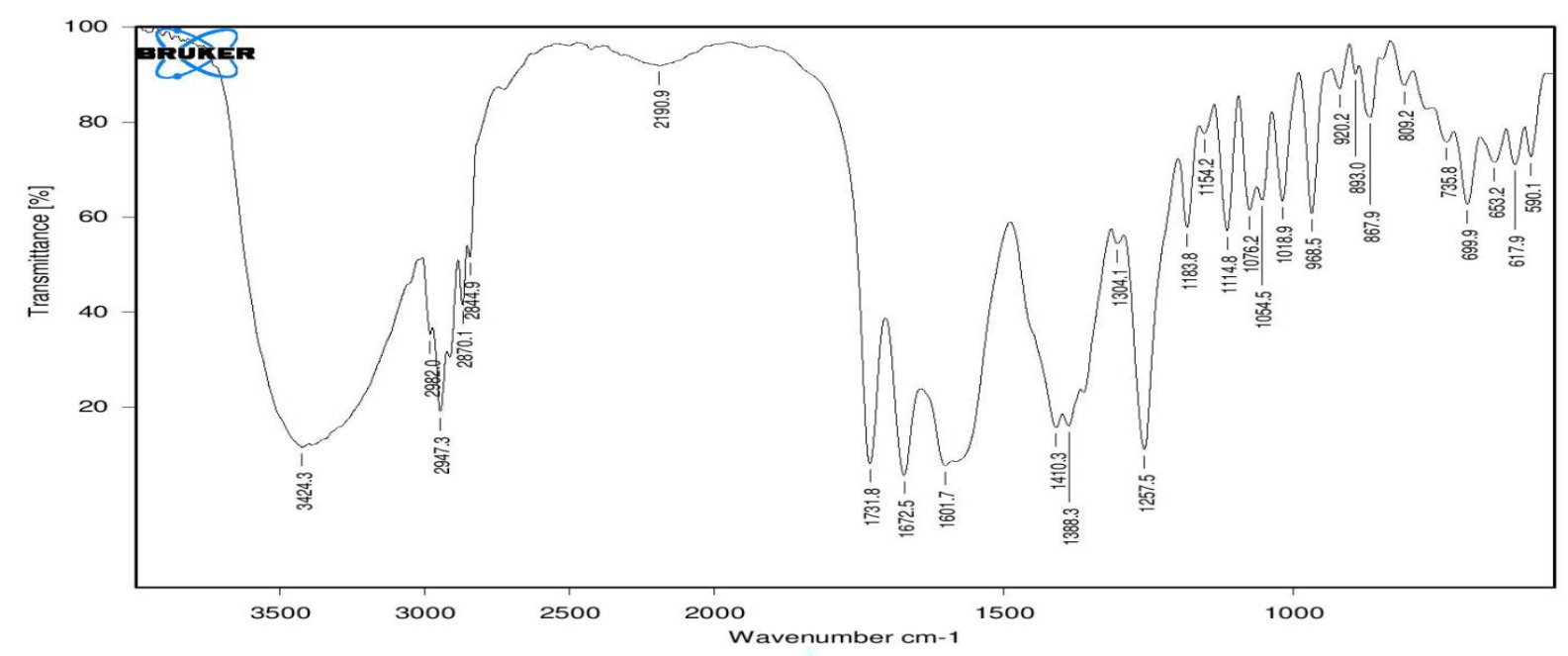

Figure 2: FTIR Spectrum of pure API

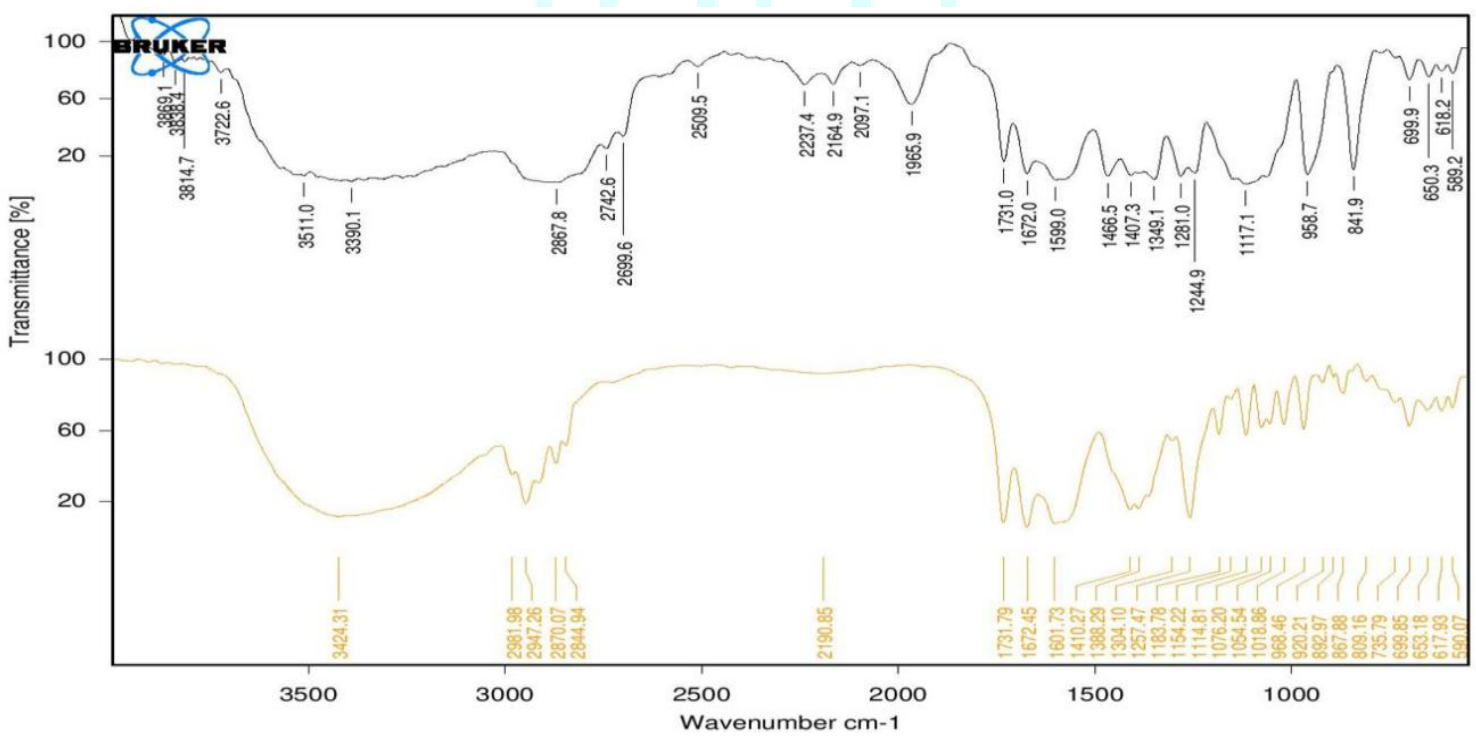

Figure 3: FTIR Spectrum of medroxy progesterone acetate with PEG 3350 


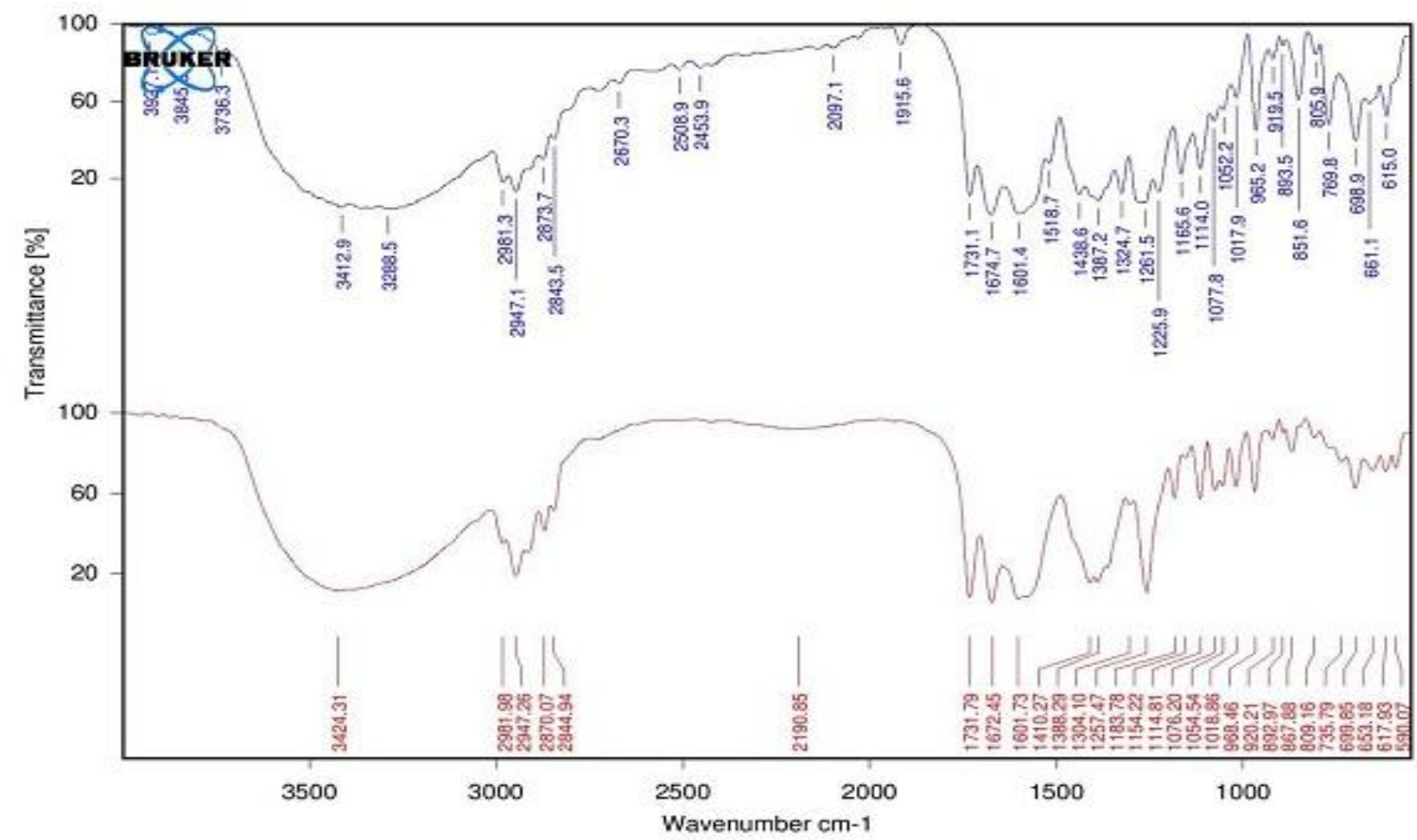

Figure 4: FTIR Spectrum of medroxy progesterone acetate with Poloxamer-188

\section{Assay}

The assay percentage of innovator and optimized formulation was $100.2 \pm 0.5$ and $99.8 \pm 0.69$ respectively. Assay was within the limit and matches with the innovator.

\section{Dissolution studies}

The drug release profiles are obtained for the innovator and different formulations F1, F2, F3, F4, F5, F6, F7, F8, and F9. Cumulative \% drug release of Optimized formulation F9 0 , $41 \pm 0.79,67 \pm 1.52,73 \pm 0.99,83 \pm 0.75,89 \pm 1.13,95 \pm 0.98$, $101 \pm 0.69$ respectively. The innovator shows the drug release $0,43 \pm 0.91,65 \pm 0.97,72 \pm 0.45,81 \pm 0.79,88 \pm 0.98,93 \pm 1$, $99 \pm 1.36$ respectively. Among all formulations, F9 trial shows the increased drug release pattern than innovator.
Table 4: Drug content

\begin{tabular}{cc}
\hline Test & Assay $(\mathbf{9 6 \% - 1 0 3 \% ~ U S P ) ~}$ \\
\hline RLD & $100.2 \pm 0.51$ \\
F1 & $98.5 \pm 1.02$ \\
F2 & $100.9 \pm 0.98$ \\
F3 & $98.7 \pm 0.59$ \\
F4 & $101.5 \pm 0.12$ \\
F5 & $97.9 \pm 0.85$ \\
F6 & $95.9 \pm 1.05$ \\
F7 & $97.8 \pm 0.39$ \\
F8 & $98.1 \pm 0.78$ \\
F9 & $99.8 \pm 0.69$ \\
\hline & Mean \pm standard deviation $(\mathrm{n}=3)$
\end{tabular}

Table 5: Cumulative Percentage drug release of profiles

\begin{tabular}{|c|c|c|c|c|c|c|c|c|}
\hline \multirow[t]{2}{*}{ Trials } & \multicolumn{8}{|c|}{ Time (hrs) } \\
\hline & $\mathbf{0}$ & 1 & 2 & 3 & 4 & 6 & 8 & 12 \\
\hline F1 & 0 & $39 \pm 0.5$ & $58 \pm 0.63$ & $67 \pm 0.20$ & $79 \pm 1.29$ & $87 \pm 0.91$ & $92 \pm 1.23$ & $96 \pm 1.49$ \\
\hline F2 & 0 & $48 \pm 0.21$ & $66 \pm 0.82$ & $72 \pm 0.85$ & $80 \pm 2.15$ & $84 \pm 0.67$ & $91 \pm 0.87$ & $97 \pm 1.54$ \\
\hline F3 & 0 & $29 \pm 0.82$ & $49 \pm 0.71$ & $65 \pm 1.09$ & $71 \pm 1.1$ & $79 \pm 0.98$ & $87 \pm 1.25$ & $93 \pm 1.49$ \\
\hline F4 & 0 & $52 \pm 1.14$ & $63 \pm 1.49$ & $71 \pm 2.1$ & $80 \pm 0.79$ & $87 \pm 1$ & $91 \pm 1.53$ & $96 \pm 1.65$ \\
\hline F5 & 0 & $35 \pm 0.75$ & $51 \pm 1.13$ & $62 \pm 0.98$ & $71 \pm 0.83$ & $83 \pm 0.8$ & $89 \pm 0.86$ & $93 \pm 0.79$ \\
\hline F6 & 0 & $27 \pm 1.05$ & $46 \pm 0.99$ & $59 \pm 1.45$ & $71 \pm 0.29$ & $87 \pm 1.23$ & $95 \pm 2.7$ & $98 \pm 1.13$ \\
\hline F7 & 0 & $50 \pm 0.91$ & $61 \pm 0.84$ & $73 \pm 1.25$ & $79 \pm 0.87$ & $86 \pm 1.4$ & $88 \pm 1.11$ & $95 \pm 2.1$ \\
\hline F8 & 0 & $32 \pm 0.85$ & $56 \pm 1$ & $69 \pm 1.05$ & $75 \pm 1.15$ & $87 \pm 1.21$ & $90 \pm 1.98$ & $94 \pm 0.94$ \\
\hline F9 & 0 & $41 \pm 0.79$ & $67 \pm 1.52$ & $73 \pm 0.99$ & $83 \pm 0.75$ & $89 \pm 1.13$ & $95 \pm 0.98$ & $101 \pm 0.69$ \\
\hline RLD & 0 & $43 \pm 0.91$ & $65 \pm 0.97$ & $72 \pm 0.45$ & $81 \pm 0.79$ & $88 \pm 0.98$ & $93 \pm 1$ & $99 \pm 1.36$ \\
\hline
\end{tabular}

Note: All the values are expressed as mean \pm SD, $n=3$ 


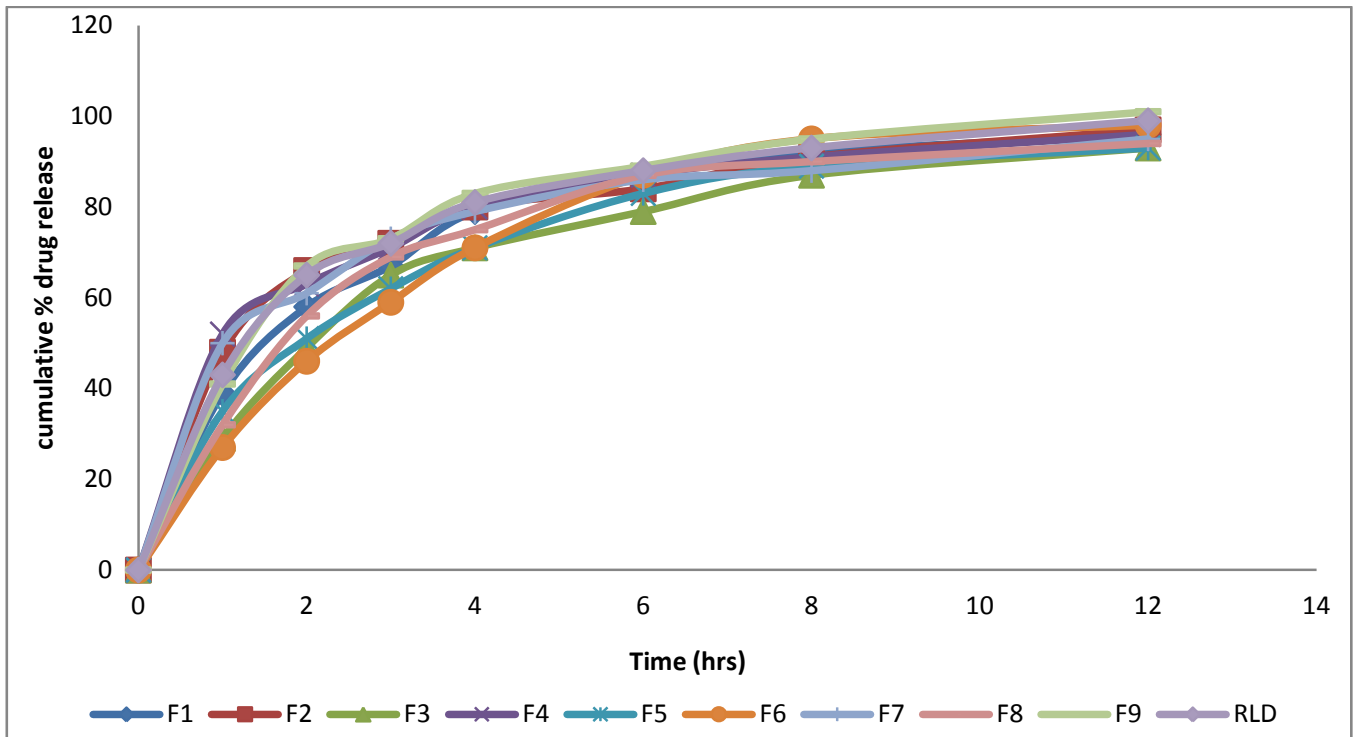

Figure 5: Cumulative \% drug release of formulations F1-F8 and RLD

Table 6: Physical characterization

\begin{tabular}{|c|c|c|c|c|c|}
\hline Trials & 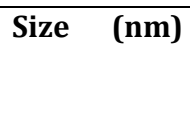 & $\begin{array}{c}\text { Zeta potential } \\
(\mathrm{mV})\end{array}$ & $\begin{array}{c}\text { pH } \\
(3.0-7.0)\end{array}$ & $\begin{array}{c}\text { Osmolality } \\
\text { (mOsmol/kg) } \\
(350-410)\end{array}$ & $\begin{array}{c}\text { Viscosity (cps) } \\
(6.0-9.0)\end{array}$ \\
\hline F1 & $1243 \pm 11.47$ & $-33.7 \pm 0.49$ & 5.67 & 375 & 6.15 \\
\hline $\mathrm{F} 2$ & $1190 \pm 32.7$ & $-21.9 \pm 3.14$ & 6.12 & 367 & 6.19 \\
\hline F3 & $1210 \pm 21.18$ & $-28.5 \pm 2.89$ & 6.67 & 364 & 6.23 \\
\hline F4 & $1240 \pm 19.8$ & $-19.8 \pm 4.1$ & 6.49 & 376 & 6.12 \\
\hline F5 & $1160 \pm 45.1$ & $-31.8 \pm 0.56$ & 5.97 & 361 & 6.51 \\
\hline F6 & $1290 \pm 12.5$ & $-29.7 \pm 2.47$ & 6.21 & 374 & 6.37 \\
\hline F7 & $1310 \pm 5.14$ & $-34.2 \pm 0.31$ & 6.60 & 361 & 6.64 \\
\hline F8 & $1180 \pm 19.2$ & $-25.9 \pm 2.73$ & 6.01 & 359 & 6.29 \\
\hline F9 & $1105 \pm 17.94$ & $-38.1 \pm 0.21$ & 6.80 & 381 & 6.95 \\
\hline RLD & $1132 \pm 7.8$ & $-35.4 \pm 0.64$ & 6.78 & 379 & 6.92 \\
\hline
\end{tabular}

Mean \pm standard deviation $(\mathrm{n}=3)$

Table 7: Sedimentation study analysis

\begin{tabular}{ccc}
\hline Time & F9 & RLD \\
\cline { 2 - 3 } (hrs) & F=Vs/Vi & F=Vs/Vi \\
1 & 1.00 & 1.00 \\
2 & 1.00 & 1.00 \\
4 & 0.99 & 0.98 \\
8 & 0.99 & 0.95 \\
24 & 0.97 & 0.91 \\
48 & 0.94 & 0.89 \\
72 & 0.90 & 0.89 \\
96 & 0.90 & 0.89 \\
120 & 0.90 & 0.89 \\
\hline
\end{tabular}

Note: Vs - Volume of sediment in $\mathrm{mL}, \mathbf{V i}$ - Initial volume \& F - Sedimentation volume

Table 8: 3M stability data of optimized formulation F9

\begin{tabular}{|c|c|c|c|c|}
\hline Formulation & F9 & F9 & RLD & RLD \\
\hline $\begin{array}{c}\text { Stability } \\
\text { conditions }\end{array}$ & $25 \div \mathrm{C} / 60 \% \mathrm{RH}$ & 40ㄷ / 75\% RH & $25 \div \mathrm{C} / 60 \% \mathrm{RH}$ & $40 \div \mathrm{C} / 75 \% \mathrm{RH}$ \\
\hline Drug content & $100.1 \pm 0.6$ & $98.8 \pm 1.23$ & $99.8 \pm 0.69$ & $98.4 \pm 1.07$ \\
\hline pH & 6.79 & 6.76 & 6.77 & 6.74 \\
\hline Osmolality & 381 & 379 & 378 & 376 \\
\hline Viscosity & 6.94 & 6.89 & 6.91 & 6.88 \\
\hline Dissolution & $101.17 \pm 0.58$ & $100.8 \pm 0.6$ & $98.87 \pm 1.63$ & $98.28 \pm 1.94$ \\
\hline Particle size (nm) & $1112 \pm 10.58$ & $1125 \pm 7.2$ & $1145 \pm 5.89$ & $1175 \pm 4.17$ \\
\hline
\end{tabular}

\section{Report:}

There were no marked changes observed in the $3 \mathrm{M}$ stability of optimized formulation F9 and innovator at 25ㅇ / $60 \% \mathrm{RH}$ ISSN: 2250-1177 and $40^{\circ} \mathrm{C} / 75 \% \mathrm{RH}$ conditions. It indicates that optimized formulation F9 shows good stability like that of innovator. 


\section{Sunitha Reddy et al}

\section{CONCLUSION}

The goal of the investigation was to develop a sustained release parenteral drug delivery system of contraceptive drug i., e medroxy progesterone acetate which was administered intramuscularly. Drug excipient compatibility was performed and the spectrums show compatibility. The formulation was optimized and evaluated for the parameters of parenteral preparations. The dissolution profile of optimized was more when compared with the innovator formulation.3M stability data at $25^{\circ} \mathrm{C} / 60 \% \mathrm{RH}$ and $40^{\circ} \mathrm{C}$ / $75 \% \mathrm{RH}$ conditions generated and it shows good stability. Hence, this product was developed to get increased compliance with decreasing the dosing frequency. A stable pharmaceutically equivalent product was developed.

\section{ACKNOWLEDGEMENT}

I am very thankful for the guidance of Dr. M. Sunitha Reddy, Asst. Prof., CPS, IST, JNTU-Hyderabad, Muhammad Fazal UI Haq and K. Anie Vijetha.

\section{REFERENCES}

1. Patel RM, Parenteral suspension: an overview, International Journal of current Pharmaceutical Research, 2010; 2(3):5-13

2. Aungst B. J, Intestinal permeation enhancers, Journal of Pharmaceutical Sciences, 2000; 89(4):428-442.
Journal of Drug Delivery \& Therapeutics. 2018; 8(6-s):298-303

3. Lawrence M. J, Microemulsion-based media as novel drug delivery systems, Advanced Drug Delivery Reviews, 2000; 45(1): 89-121.

4. Patel CA, Keraliya R, A review: Parenteral depot drug delivery system, Journal of Drug Delivery Research, 2014; 3(1):1-10.

5. Parrott E L, Pharmaceutical technology: fundamental pharmaceutics, Burgness Publishing Company; 1970.

6. Cundy T, Farqubar CM, Cornish J, Reid IR, Short-term effects of high dose oral medroxy progesterone acetate on bone density in premenopausal women, Journal of Clinical Endocrinology and Metabolism, 1995; 81(3):1014-1017.

7. Chaurasia G, A review on pharmaceutical preformulation studies in formulation and development of new drug molecules, International Journal of Pharmaceutical Sciences and Research, 2016; 7(6):2313-2320.

8. Alam. A, Alka Ahuja, Sanjula Baboota, S. K. Gidwani, J. Ali, Formulation and evaluation of pharmaceutically equivalent parenteral depot suspension of methyl prednisolone acetate, Indian Journal of Pharmaceutical Sciences, 2009; 71(1):30-34.

9. William P. Forrest, Kevin G. Reuter, Vivek Shah, Irina Kazakevich, Michael Heslinga, Siddhi Dudhat, Sanjaykumar Patel, Claudia Neri, Yun Mao, USP Apparatus 4: A Valuable In Vitro Tool to Enable Formulation Development of Long-Acting Parenteral (LAP) Nanosuspension Formulations of Poorly Water-Soluble Compounds, AAPS PharmSciTech, 2017; 19(1):413-424.

10. Raymond C.R., Paul J.S., Marian E.Q. Handbook of Pharmaceutical excipients. Sixth edition. U.K. 